\title{
The importance of physical function to people with osteoporosis
}

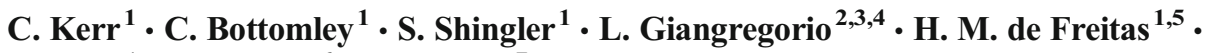

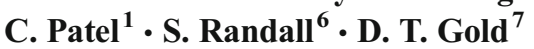

Received: 8 August 2016 / Accepted: 3 January 2017 / Published online: 6 March 2017

(C) The Author(s) 2017. This article is published with open access at Springerlink.com

\begin{abstract}
Summary There is increasing need to understand patient outcomes in osteoporosis. This article discusses that fracture in osteoporosis can lead to a cycle of impairment, driven by complex psychosocial factors, having a profound impact on physical function/activity which accumulates over time. More information is required on how treatments impact physical function. Introduction There is increasing need to understand patientcentred outcomes in osteoporosis (OP) clinical research and
\end{abstract}

Catherine Bottomley, Cicely Kerr, Sarah Shingler, Hayley M. de Freitas, and Chloe Patel were employees of ICON at the time the research was completed.

Electronic supplementary material The online version of this article (doi:10.1007/s00198-017-3911-9) contains supplementary material, which is available to authorized users.

H. M. de Freitas

hdefreitas@mapigroup.com

1 ICON Patient Reported Outcomes, W. Diamond Avenue, Suite 1000, Gaithersburg, MD 20878, USA

2 University of Waterloo, 200 University Avenue West, Waterloo, ON N2L 3G1, Canada

3 Geriatric Education and Research in Aging Sciences Centre, Hamilton, ON, Canada

4 Research Institute for Aging, Waterloo, ON, Canada

5 Mapi, Translation and Innovation Hub Building, 80 Wood Lane, White City, London W12 0BZ, UK

6 National Osteoporosis Foundation, 251 18th Street South, Suite 630, Arlington, VA 22202, USA

7 Duke University Medical Center, Durham, NC 27710, USA management. This multi-method paper provides insight on the effect of $\mathrm{OP}$ on patients' physical function and everyday activity. Methods Data were collected from three sources: (1) targeted literature review on OP and physical function, conducted in MEDLINE, Embase and PsycINFO; (2) secondary thematic analysis of transcripts from patient interviews, conducted to develop a patient-reported outcome instrument. Transcripts were re-coded to focus on OP impact on daily activities and physical function for those with and without fracture history; and (3) discussions of the literature review and secondary qualitative analysis results with three clinical experts to review and interpret the importance and implications of the findings. Results Results suggest that OP, particularly with fracture, can have profound impacts on physical function/activity. These impacts accumulate over time through a cycle of impairment, as fracture leads to longer term detriments in physical function, including loss of muscle, activity avoidance and reduced physical capacity, which in turn leads to greater risk of fracture and potential for further physical restrictions. The cycle of impairment is complex, as other physical, psychosocial and treatment-related factors, such as comorbidities, fears and beliefs about physical activity and fracture risk influence physical function and everyday activity.

Conclusion More information on how treatments impact physical function would benefit healthcare professionals and persons with $\mathrm{OP}$ in making treatment decisions and improving treatment compliance/persistence, as these impacts may be more salient to patients than fracture incidence.

Keywords Health-related quality of life - Osteoporosis · Patient-centred outcomes · Physical function · Psychological outcomes $\cdot$ Social outcomes 


\section{Introduction}

Osteoporosis (OP) is a growing public health concern and affects more than 200 million people worldwide [1]. Individuals living with $\mathrm{OP}$ are at greater risk of experiencing a fragility fracture. In developed countries, lifetime risk for a wrist, hip or vertebral fracture has been estimated at 30-40\% [2]. For individuals aged 50 years and over, approximately one in two women and up to one in four men will experience an osteoporotic fracture [3]. Worldwide, the disease has been reported to account for more than 8.9 million fractures annually [4]. Due to the importance of fracture in the progression of OP, clinical trials investigating novel therapeutic agents have focused on endpoints relating to bone health and fracture incidence. The US Food and Drug Administration (FDA) approval requirements for new therapies emphasise reducing the risk of vertebral fracture along with maximising drug safety [5].

While fracture incidence is clearly of clinical importance in OP, the extent to which it sufficiently captures outcomes that are patient-centred remains unclear, particularly given the potential impact of fracture on patients' everyday lives such as mobility and other types of physical activity. Initially OP is considered asymptomatic, but this situation can change dramatically once a fracture has occurred. Fractures can result in reduced mobility and physical function. They can also result in chronic pain or affect the ability to self-care. Fractures can also have long-term consequences on physical function and greatly affect patients' health-related quality of life (HRQL) [6-9].

The importance of patient-centred outcomes when assessing new treatments is being recognised across a range of therapeutic areas. The FDA, along with the Patient-Centered Outcomes Research Institute (PCORI), has published guidance on the use of patient-reported outcomes (PROs) in product labelling and clinical research, respectively, and highlighted their value [10, 11]. A greater awareness of patient-centred outcomes has the potential to guide treatment decisions and encourage more patient-focused prescribing. Nevertheless, PROs are assessed infrequently in OP clinical trials and when included, rarely involve more than a small subset of subjects. As a result, any opportunity to detect a meaningful change in PROs related to treatment is limited. Furthermore, in instances where benefits in PROs have been detected during well-controlled trials [12-15], these outcomes have been omitted from product labels. As such, there is an increasing need to understand and capture patientcentred outcomes in OP. The objective of this paper is to use evidence and patient perspectives to provide insight on the effect of OP on patients' physical function.

\section{Methods}

A multi-method approach was used to consolidate our understanding of physical function in OP. A systematic search and narrative literature review was conducted, to seek and summarise published evidence related to daily activities requiring physical function in OP. In addition, a secondary qualitative analysis of patient data, originally collected for the development of a PRO instrument, was performed. Finally, input was sought from key clinical thought leaders specialising in OP, to review, interpret and add to the findings of the literature review and interview analysis. The final result is a comprehensive perspective on the impact of OP and osteoporotic fragility fractures on physical function or performance.

\section{Literature review}

The literature search was designed to identify publications describing the impact of OP on daily activities and physical function. The search was conducted in MEDLINE and Embase using the web-based platform Ovid. Developing the search strategy was an iterative process with the initial use of draft searches, checking the outcome by reviewing a proportion of resulting abstracts for relevance and updating the search terms to improve the relevance of results. The initial search strategy included terms focused on OP and daily activities and function and was limited to more recent publications (published within 8 years) and those including patients aged $\geq 45$ years. A further search strategy was developed to specifically identify qualitative studies that discussed the impact of OP on physical function and daily living from a patient's perspective and was conducted in PsycINFO, in addition to MEDLINE and Embase. The final search terms are presented as a supplementary appendix. Abstracts were reviewed and those deemed relevant were selected for full review. Articles were deemed relevant if they reported on the impact of OP on physical function/daily activities or the relationship between physical function and fracture occurrence.

\section{Secondary qualitative analysis of interview transcripts}

Given the lack of existing literature around OP and physical function from the patient perspective, a secondary thematic analysis of data collected during interviews with people with OP was performed. These interviews were originally conducted during the development of the Osteoporosis Assessment Questionnaire-Physical Function (OPAQ-PF) questionnaire, a PRO instrument developed to evaluate osteoporosis treatment effectiveness [16]. During the instrument development process, 39 people with OP were recruited from three clinical centres in the USA, and concept elicitation and cognitive debriefing interviews were conducted. All patients were consented to the study, all interviews were conducted using a semistructured interview guide in a face-to-face setting by an experienced, trained interviewer and all were audio-recorded and transcribed. The sample was all female with a mean age of 70 years (standard deviation $[\mathrm{SD}]=10.35$ ), and the majority 
were white $(n=34)$. All participants had been living with $\mathrm{OP}$ for at least 1 year, and the mean disease duration was 7.2 years $(\mathrm{SD}=2.16)$. The majority of participants $(n=27)$ had experienced one or more osteoporotic fractures, with most having had a single fracture $(n=22)$. The maximum number of fractures experienced by a single participant was three. Fractures had occurred between 3 and 140 months (11.6 years) prior to being interviewed, with a mean time since fracture of 31.6 months $(\mathrm{SD}=37.9)$. Participants with comorbidities were not excluded from the sample in order to reflect the wider OP population, as comorbid conditions are very common in this population. However, this made it more difficult in some cases to attribute impact on physical function to OP. Comorbidities included osteoarthritis, rheumatoid arthritis, diabetes, asthma and chronic obstructive pulmonary disease (COPD).

The secondary, thematic analysis of the interview data was conducted in two phases; the first was at a broad level to isolate relevant sections of the transcripts (i.e., those that focus on physical impacts of OP including any discussion of physical functioning, physical activities of daily living and the importance to patients of this type of impact). Data analyses were conducted by two of the authors (SS and CP) using MAXQDA 10, a qualitative analysis software tool that facilitates systematic coding. The two researchers (SS and CP) familiarised themselves with the data and generated initial codes on the same two transcripts. The initial codes were then compared, to check for consistency, ensuring that both researchers were including the same/very similar sections for further coding. The following stage of analysis involved coding the relevant sections in greater detail that related to OP impact on daily activities and physical function, the relative experience and impact of patients with osteoporotic fracture versus those who had not had osteoporotic fracture, and the importance of these impacts to patients. The codes identified during analysis were then grouped into themes which were then reviewed and defined by the researchers (SS and CP).

\section{Review by clinical experts}

Results from the literature review and secondary qualitative analysis of interview transcripts were reviewed and discussed with three clinical experts (LG, SR and DTG) specialising in OP with extensive experience in investigating HRQL, medication compliance and persistence, physical activity, exercise recommendations and OP interventions, through academic research, clinical practice or patient advocacy. Discussion took place during two in-depth telephone conferences, held on the 8th and 23rd May 2014. Specific questions considered in these discussions are presented in Table 1. Further input was sought through
Table 1 Specific questions considered during discussions with OP experts

- What effect does decline in physical function have on the daily lives of people with OP?

-What is the value of improvement of physical function and ability to perform daily activities requiring physical function to people with OP?

- What is the value of maintaining physical function and the ability to perform daily activities requiring physical function to people with OP?

- How strong is the relationship between fracture occurrence and ability to perform daily activities requiring physical function in OP? To what extent does this vary between individuals?

- How much of the decline in physical function experienced by people with OP is not explained by fracture occurrence?

- What other factors affect physical function and performance of daily activities requiring physical function in people with OP?

- What are the unmet needs or gaps in current treatment in relation to the effect of $\mathrm{OP}$ on peoples' performance of daily activities requiring physical function?

- What is the added value of evaluating physical function to inform treatment effectiveness? What does measurement of physical function capture that is not already explained by fracture occurrence?

an iterative and collaborative process of developing a written summary of the discussions.

The information gathered in the literature review, secondary qualitative analysis of patient interview transcripts and clinical input were consolidated and grouped by common themes. Quotes from patient interview transcripts are included to illustrate points from the perspective of people living with OP. Each quote is followed by details of the individual to link to the original data source (identification number) and provide contextual information to aid interpretation (their age, the number and location of fractures they have experienced, the length of time since fracture(s) in months and any comorbid conditions). Where relevant, the interviewer's questions are stated in italics.

\section{Results}

The examination of findings from the three data sources suggests that overall, osteoporosis and osteoporotic fragility fractures can have a substantial impact on physical function and this impact accumulates over time, through a cycle of physical impairment, limitation and restriction. This cycle of impairment is illustrated in Fig. 1 and depicts the relationships among fracture, physical impairment, activity restrictions and loss of bone/muscle strength over time. It also documents the role of added complexities, such as psychological and social factors and comorbid conditions, in the further limitation of physical function and everyday activity. Using the cycle of impairment as a framework, results on the impact of OP and fracture on physical function are detailed in the following sections 
Fig. 1 The cycle of impairment and fracture in $\mathrm{OP}$

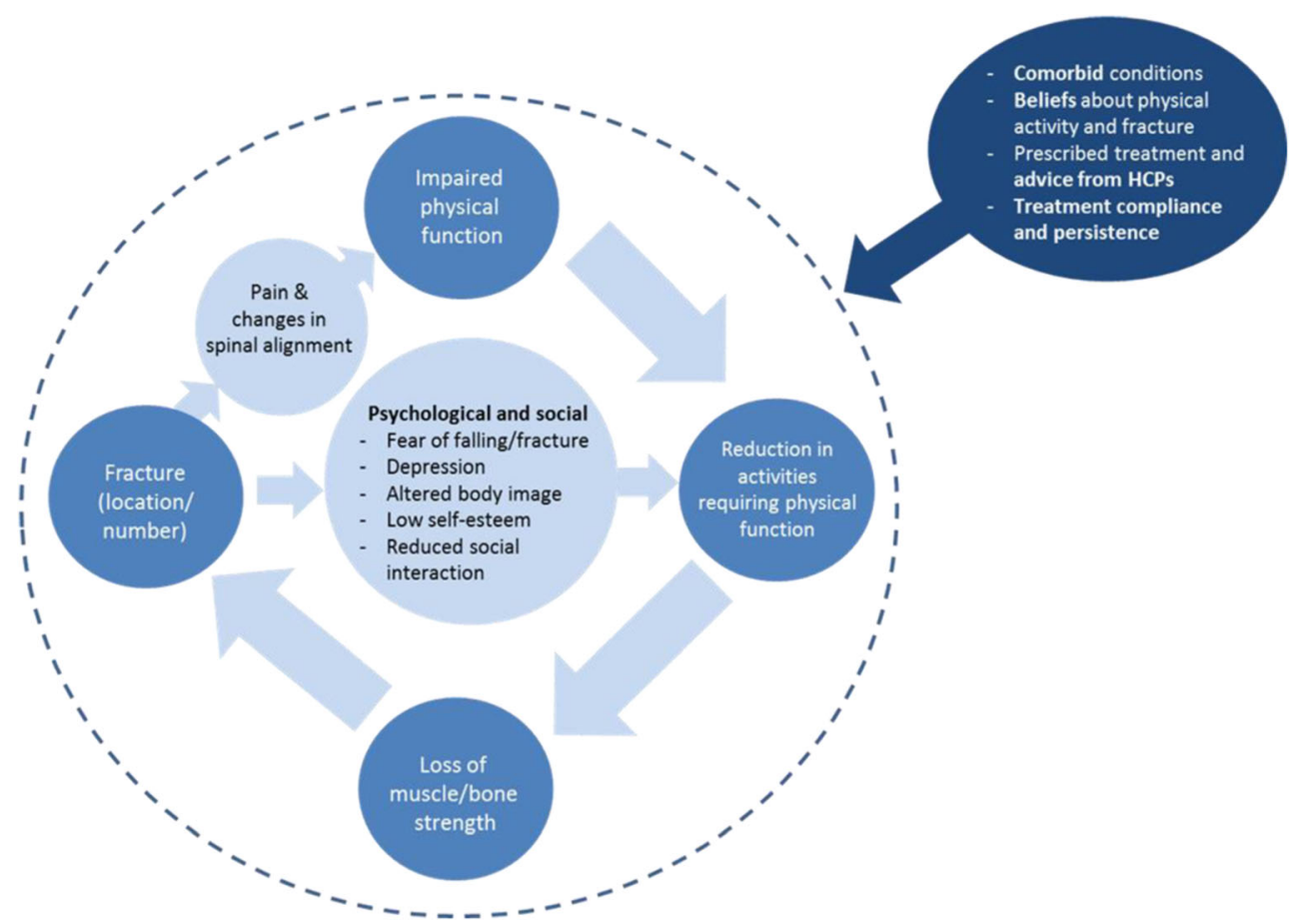

based on the findings from the literature, secondary analysis of patient interview data and clinical expert discussions.

\section{Physical function in OP and reduced activity}

Physical function is an individual's ability to perform physical tasks and activities and is known to be affected in OP, among those both with and without fracture [16]. Impairment of physical function in OP manifests as an inability to complete regular, daily activities requiring physical movement and strength. Affected activities include those requiring mobility (such as walking, climbing stairs), certain physical positions (such as sitting, picking things up from the floor) and physical transfers (such as getting in or out of a chair, on or off the toilet).

“...taking care of personal hygiene, it just-you just have to move a little slower and take your time because you're limited in your range of motion... if you're washing your back or something or trying to wash your hair, you're limited, so it seems like it takes forever and therefore, you get, kind of, tired" [R1P3, 55 years, one fracture, 36 months since fracture.]

People with OP report being unable to carry out these activities unaided even after fractures have healed, and often require help to carry out basic daily activities [16]. Numerous factors are known to influence physical function in OP, most notably the occurrence of fractures and the loss of bone and muscle strength.

\section{Fracture occurrence}

The occurrence of fracture(s) is known to cause increasing limitations in physical function for people with OP. Various tools (e.g. the Continuous Scale Physical Functional Performance [CS-PFP] and the Physical Performance Test $[\mathrm{PPT}])$ are available to assess the usual functional movements in older adults $[17,18]$. Physical performance is measured during standardised functional tasks in terms of time taken to complete a task, weight lifted and/or distance covered. The Safe Functional Motion Test (SFM) [19] builds on the constructs and tasks of the CS-PFP and PPT and is specifically designed for use in people with osteoporosis. The SFM quantifies physical function in terms of body mechanics and movement strategies used to perform everyday activities [19]. Using the SFM, it has been shown that those with hip or vertebral fracture and fall history are more likely to have poor performance and physical function when completing everyday tasks than those with no history of fracture or falls [20]. Analysis of an osteoporosis clinic database indicated that patients with fewer limitations in physical performance according to SFM scores were less likely to present with vertebral compression fracture (odds ratio [OR] 0.89; $p=0.036$ ), prevalent hip fracture $(\mathrm{OR}=0.77 ; p=0.004)$, or a history of injurious fall $(\mathrm{OR}=0.80 ; p=0.003)$ after adjusting for other important covariates [20].

Outcomes after a fracture may be worse among older people for whom poor physical health is likely to be associated with social disconnectedness and perceived isolation [21]. The influence of fracture on physical function and other 
aspects of individual well-being is likely to vary depending on (i) fracture location and (ii) the number of fractures experienced.

\section{Fracture location}

Fractures of the wrist or vertebra are perceived to result in fewer physical impairments or restrictions than hip fracture during the acute stages. The influence of fracture on physical function will also depend upon the degree of pain and recovery trajectory associated with fractures, which will vary by fracture type and severity. Wrist fracture may still affect daily activities requiring physical function, particularly due to reduced grip strength during and after healing. The effects of spine fracture on physical function may be quite variable, depending on the number, severity and chronicity of fracture(s), or the presence of pain. A hip fracture is immediately debilitating, limits mobility and results in hospitalisation, with physical function impairments that can often persist long after the fracture. Importantly, mobility impairments due to hip fracture may restrict participation in life roles or the capacity to live independently.

"I was on my feet for three hours making dinner and I love cooking, but since I'm so slow anymore, you know, it took an extra hour than it normally would and my back and my upper hips were feeling it. They were hurting" [P20, 57 years, two fractures at the hip and femoral shaft, timing unknown, comorbid osteoarthritis and diabetes]

“...I'd kind of get a little bit too tired and it starts hurting my wrists and stuff when I dig stuff or do things heavily, you know" [P17, 74 years, two fractures at the forearm and foot, 8 months since both fractures, comorbid osteoarthritis]

The relationship between fractures and physical function is particularly important in the case of asymptomatic vertebral fractures, which do not cause clinical manifestations, such as pain or obvious limitation. People with asymptomatic fractures are unlikely to seek medical advice and often continue with their lives, accommodating and adapting to their reduced strength or speed but also restricting their physical activity, for an extended period of time. Vertebral fractures are globally under-recognised, under-reported and under-managed in people with OP [22]. Furthermore, a single vertebral fracture increases the risk of a future fracture by five and doubles the risk of a future hip fracture [23], with asymptomatic fractures carrying the same risk as those that have been diagnosed.

Number of fractures The number of fractures the person with $\mathrm{OP}$ has experienced is negatively correlated with physical function and HRQL [6-9]. Many studies use the presence of one or more fractures as a meaningful cut-off to predict lower HRQL, but it is likely that physical and psychological impairments increase with increasing number of fractures [14, 24]. Individuals with multiple fractures may experience: reduced speed in performing activities requiring physical function, reduced strength, hyperkyphotic posture and suboptimal vertebral alignment, and reduced mobility $[25,26]$.

"I couldn't do anything with the-at my Pilates class 'cause, you know, my foot hurt. I couldn't put my foot to the actual ground, so if you-without the shoe, there was no way I could walk, so it was pretty painful, so it affected everything". [P4, 60 years, two fractures at the shoulder and toe, 22 and 6 months since fractures]

In addition, outcomes such as hyperkyphosis also lead to increased mortality and a higher risk of future fractures [27, 28]. Multiple fractures affect biomechanics, HRQL, fear, body image as well as physical function. People who have experienced only a single vertebral fracture (and no other osteoporotic fractures), commonly report little effect on daily activities requiring physical function. However, this can change significantly once an individual has experienced two or more fractures, particularly where they cause pain or hyperkyphosis.

\section{Loss of bone and muscle strength}

Loss of bone and muscle strength are often experienced together, especially in older individuals [29, 30]. Research has shown an association between muscle strength and bone health [30-32], with the various facets of muscle strength (e.g. mass, function and performance) generally showing a positive relationship with bone mass or density. Muscle weakness experienced by those with OP may also be due to physical de-conditioning from reduced activity levels, where people with OP stop or severely restrict daily activities and other exercise. The degree of muscle loss can increase following fracture, particularly if the fracture results in pain, which can limit activity, such as after a vertebral fracture, or after long period of inactivity, such as after a hip fracture.

"Does my fracture have an impact on my ability to climb steps or stairs, and as I said before, some of it comes directly from pain that might be caused from the fracture, but a lot of it has to come from the effects on my body since the fracture that if you're not staying in shape, then you're losing endurance, you're losing muscles and you're not able to complete some simple tasks just because of that. So it's, kind of, a secondary effect from the fracture". [P21, 59 years, one fracture at the spine, 11 months since fracture] 
The term sarcopenia has been used to define various aspects of muscle health loss, but more recently, it has been defined as a loss of muscle mass and function [29]. OP and sarcopenia are common in older age and associated with significant morbidity and mortality [29]. When people with OP also demonstrate the criteria for sarcopenia, they and those around them can become concerned about their ability to perform daily activities safely and without assistance. Fractures or weakness (perceived or real by person with OP or family/ caregivers) may lead to activity avoidance or limitation.

\section{Psychological and social factors related to physical impairment/reduced activity}

Individuals with OP may experience fear (particularly of falling or fracture), depression, and other psychological outcomes. These consequences are often underestimated when considering the overall burden of OP [33]. The psychological effect of an OP diagnosis can be increased where people anticipate change in body shape and physical decline. Due to the hereditary and age-related aspects of OP, people diagnosed with OP may already have observed hyperkyphosis, other changes in body shape or decline following fractures in their relatives or peers, even where this may not have been confirmed by OP diagnosis. Psychological outcomes can add to limitations in physical function to reduce social activity, interaction and participation. Social and disease-specific support groups may have a positive impact on psychological and social outcomes in OP [34].

\section{Fear/anxiety}

People with OP report caution and fear of fracture during daily activities requiring physical function and physical activity [35]. For example, a quality of life survey of 234 female patients with persistent pain due to vertebral fracture found that fear of more fractures or falling was correlated to the domains of physical limitations $(r=0.43 ; p<0.001)$, activities of daily living $(r=0.44 ; p<0.001)$ and leisure $(r=0.25 ; p<0.05)$ [35]. Fear can be further exacerbated by reduced muscle strength and a lack of confidence in strength to safely perform certain activities. As a result, activities are completed more slowly, less often or avoided altogether.

"Yeah, I stopped riding my bike too 'cause I'm afraid of falling" [P11, 74 years, one fracture at the ribs, 12 months since fracture, comorbid osteoarthritis]

“...I don't accomplish all the things I wanted to do and I find myself sitting more. And I did go to water aerobics, but now I told the Physical Therapist that if I go to water aerobics now, if I drive over there, then when I get in the pool, the steps are very steep, the steps are big and I'm afraid I can't get out and I don't have the strength to pull myself out of the pool. So I mean, it limits me to so many things that I did before". [P18, 84 years, comorbid osteoarthritis]

Fear of falling and impaired physical performance are also related to intensity of back pain in people with $\mathrm{OP}$, irrespective of vertebral fracture and fall history [36]. Other psychosocial consequences of OP such as frustration and anxiety can also contribute to reduced activity levels, whereas regular exercise can actually reduce symptoms of anxiety [37].

\section{Depression}

Many people with an osteoporotic fracture experience either situational or chronic depression, and regular pain is known to be an important factor in psychological well-being [38]. Preliminary data from the CORE study indicated a high prevalence of depression $(69 \%)$ in osteoporotic-fragile elderly females (mean age 85 years) [39]. A large nationwide population-based study based on the Taiwan Longitudinal Health Insurance Database found that the incidences of major depression were $1.2 \%$ in those hospitalised for a femoral neck fracture and $0.7 \%$ in those in the non-fracture comparison group (hazard ratio [HR] 1.82; $95 \%$ confidence interval [CI] 1.30, 2.53; $p=0.001$ ) [40].

Depression can reduce both physical energy and psychological motivation to carry out daily activities requiring physical function. Therefore, it may prevent people from increasing their physical function when advised to do so. Depression can also lead to social isolation and affect cognitive abilities, which can result in restricted participation in social activities or life roles [39-42].

\section{Altered body image}

OP and fracture(s) can cause a change in body image which can have a significant effect on physical and social functioning. Older adults can lose up to 1 inch of height as they age, but the effect of osteoporotic fractures on height loss and deformity can be substantially worse. Vertebral fractures change both the shape and alignment of the lumbar and thoracic spine, leading to hyperkyphosis or "dowager's hump". Other changes can include a constriction of internal organs, protruding abdomen, forward head position and flattening of the lordosis or curve in the lower back. These significant changes substantially alter an individual's appearance and result in ill-fitting clothes.

The issue of self-image and clothing was found to be highly important during the Choices ${ }^{\mathrm{TM}}$ patient education programme for OP $[43,44]$. In addition, the need for a supportive device such as a cane or walker further enforces an individual's selfconsciousness of impairment. A negative body image is likely to influence an individual's willingness to participate in social 
activities and hence lead to social isolation. Furthermore, people can be unwilling to use recommended mobility aids in order to avoid negative perceptions from others. By not using such aids, people can increase their risk of falls and fractures thus leading to a possible decrease in physical function.

\section{Low self-esteem}

Clinical experience suggests that the low self-esteem experienced by people with OP can result from a number of factors, which include an increasing inability to continue, perform or engage in previously normal activities. Decrements in physical function often result in a shift in family or societal roles. For example, women may be told or feel they are no longer able to pick up more than 10 or $15 \mathrm{lb}$, leading to a change in their grand-parenting role and the inability to interact with a grandchild.

"Before I was diagnosed, I would pick up my grandkids, hold them and were 50,60 pounds ... since the diagnosis, I'm just much more careful. I'm like, 'No, I'm not going to do that', you know". [P6, 57 years]

Other functional limitations may lead to a reduced ability to complete previous volunteer or work roles. In addition, people with OP may experience reduced self-worth when they compare themselves with others their age that are able to continue to carry out these roles. Low self-esteem can also be linked to body image and people with OP often worry about how they are seen by others, particularly when hyperkyphosis leads to visible changes in physical appearance. Low self-esteem can also be caused by self-blame for having the condition; for example, feeling they may have done something to cause their $\mathrm{OP}$, such as not eating a healthy diet or exercising enough.

\section{Comorbid conditions}

Comorbid conditions are common and variable in the OP population as was found in the interview sample, and include other musculoskeletal conditions and autoimmune disorders [45]. The presence of comorbidities can affect the ability of people with OP to complete daily activities requiring physical function. Any comorbid condition, especially arthritis, can exaggerate the effects of OP, potentially making daily activities requiring physical function harder.

"I mean, as you get older with this bending, sometimes I'll bend down and I think, oh, I can't get up, I mean, you know, yes, so there is some problems, but I don't know if it's related to osteoporosis or just the arthritis and aging, you know, so it's hard to know". [P11, 74 years, 1 fracture at the ribs, 12 months since fracture, comorbid osteoarthritis]
Some comorbidities can increase the risk of fracture, particularly when the risk of falls is increased [46]. Symptoms such as neuropathy, orthostatic hypotension and dizziness which increase the risk of falling can also predict the risk of fracture and decline in physical activity. In addition, comorbidities which cause pain, for example rheumatoid arthritis, can alter peoples' level of physical activity and change the way they carry out daily activities requiring physical function, leading to an increased risk of fracture. As a result, assessing functional impairment caused by OP can be difficult when other chronic conditions are present and this was evident in the interviews. Osteoarthritis and OP are often confused by patients, who may not understand the difference between these two conditions or what disease caused which symptoms.

“There isn't a day that I don't feel the osteoporosis in my hands, in my back, in my legs, in my toes and, you know, whether I'm bending, or whether I'm walking, or whether I'm stepping down, getting off the bed, going to the bathroom, standing back up, even going- sitting on the john..." [P13, 82 years, comorbid osteoarthritis]

\section{Beliefs about physical activity and fracture}

Perceptions and beliefs around physical activity are important influences of physical function. If people with $\mathrm{OP}$ are not used to being physically active or undertaking exercise, then a diagnosis of OP may not change this, even if advice on the types and amount of exercise recommended is provided. Some people with OP may choose to become inactive as a result of the diagnosis because of an increased fear of falling or fracture and increased fear may make it difficult to engage some people with OP in exercise programs.

"Well I'm supposed to do a lot of walking, which I know I can do for osteoporosis, but the sidewalk is uneven and it's dangerous. I have to be careful. I have to use a walker where I normally wouldn't" [P10, 61 years, comorbid Parkinson's disease]

For those unused to being physically active before OP, cultural factors and other learned behaviour can represent additional barriers to adopting a more physically active lifestyle [47]. Conversely, people who exercise regularly and consider themselves "healthy" can incorrectly attribute low-energy fractures to external events rather than bone health, believing that their preventative behaviours or absence of other risk factors means they are at low risk of future fracture [48, 49]. Perceptions around the consequences of fracture are important. Research has shown that women, who blame fractures on external events and do not attribute them to bone health, can experience long-term consequences for personal health, social 
and financial implications, which are often underestimated [50].

In individuals with osteoporosis, differences in levels of self-efficacy (the belief that one can carry out a behaviour) may have personal health, social and financial implications. Self-efficacy may impact an individual's willingness to adopt a more active lifestyle, to eat healthily or up take of treatment for osteoporosis. This impact should be a consideration for researchers, as exemplified in a recent Maternal Vitamin D Osteoporosis Study (MAVIDOS) in which mothers with higher self-efficacy were less likely to experience practical problems taking vitamin $\mathrm{D}$ during the trial [51].

\section{Prescribed treatment and advice from healthcare professionals}

Exercise advice from physicians and other providers without training in physical activity is often too general to be useful. For example, a common recommendation made by physicians to people with OP is not to lift "anything heavier than a 5 pound bag of sugar".

\section{"Tell me about how osteoporosis impacts you on a day- to-day basis?}

Hmmm. Well, it does-I am on restrictions now.

Okay and what are those restrictions?

It's not lifting more than ten pounds". [P14, 66 years, one fracture at the spine, 7 months since fracture]

This kind of nonspecific general advice is not helpful to many people with OP, who can experience a fracture when completing activities that do not involve lifting heavy objects, for example bending over to tie their shoes. In addition, people with OP may be advised by their physicians to stop the leisure activities that they enjoy and those which get them out of the house and exercising. Golf, skiing, yoga and Pilates are forms of physical activity that professionals often advise against because of concerns about increased risk of fracture. However, applying advice to avoid certain activities is appropriate in other instances, such as sit ups or abdominal curls or rotations, as these and similar activities involve twisting of the spine or "flexion" which may cause vertebral fractures [52]. However, it is important to tailor advice according to risk, activity history and other factors, such as time since fracture or use of osteoporosis medication. Further, one might consider that placing restrictions is a disincentive to physical activity and may create fear, whereas advice on how to move safely and modify activities may provide an incentive to stay active. For example, some people with osteoporosis (e.g. otherwise healthy, but at moderate risk of future fracture) may simply need to practice 'spine sparing' strategies and take efforts to reduce fall risk [53].
Advice provided by physicians has influence beyond the physical well-being of the patient, and ceasing participation in leisure activities and hobbies is also likely to affect social and psychological outcomes. People with OP have reported that they view exercise as an important way of taking an active role in their health and feel that being diagnosed with OP means they are unable to participate in fun, socially engaging exercise activities and are instead advised by healthcare professionals to undertake less enjoyable structured exercise programs. Multimodal exercise has been identified by experts as a priority intervention in $\mathrm{OP}$ and shown to reduce pain and improve quality of life in individuals with vertebral fractures $[53,54]$.

\section{Treatment compliance and persistence}

Poor compliance and persistence with OP medication and other interventions can result in increased fracture risk, pain and subsequently further limitations in physical function. Reasons for noncompliance and nonpersistence to therapy include cost, inconvenience, safety issues and efficacy concerns of osteoporosis medications. When patients are asked to rank these attributes of OP medication, their age, education level, income and experience of prior fractures have been shown to have a significant effect on their treatment decision [55]. Patient engagement and interest in treatment are a key driver of compliance and persistence, and poor compliance and persistence with OP medication have been shown to be mostly due to deliberate choice rather than forgetfulness [56]. There is also little knowledge about the prevalence of primary noncompliance and nonpersistence with these medications.

In OP, patient engagement in treatment can be especially challenging if it involves increasing exercise beyond usual levels, and this is particularly problematic in elderly individuals. Long-term compliance and persistence with exercise interventions among individuals with OP are variable and can range from 39 to $95 \%$ [57-59]. Older adults with OP report not being interested in exercise, being in too much pain to exercise and having a fear of falling [60].

\section{Discussion}

While capturing fracture incidence is well-recognised as important in the study and management of OP, there is an increasing need to understand the physical impact of OP beyond fracture to ensure patient-centred research and management. The cycle of impairment presented here summarises how numerous factors can contribute to an increasing limited physical function in people with OP. Firstly, limiting activity can contribute to loss of muscle and bone strength and subsequently an increase in fracture risk. In turn, fracture occurrence can result in pain, fear of falling or changes in alignment, which 
can limit the performance of daily activities requiring physical function. Thus, a decrease in physical function can lead to an accumulation of impairment and activity restrictions over time.

Factors affecting outcomes across the physical, social and psychological spectrums are interrelated, and further elucidating these interactions is important to understand the true consequences of OP. The confounding effects of comorbidities and poor treatment compliance and persistence also must not be overlooked. This multifactorial picture of OP has important implications for treatment, as only once we understand all the factors that may influence an individual's disease can we truly provide efficacious treatment. Limitations in physical function and restricted participation in society can occur gradually and are influenced by a range of factors including pain, availability of social support and the number of fractures. The situation can be made worse where vertebral fractures are not diagnosed or where advice from healthcare professionals is not based on validated assessment or sufficiently tailored to the individual.

The results of this review support that patient-centred outcomes are important to capture for evaluating treatment and managing the condition, as they provide additional insight into the impact of OP and fracture on the patient's everyday life and overall well-being, improvement in which are key goals of treatment. Currently, few studies have examined the effect of pharmacologic treatment on physical function outcomes in OP, yet understanding the benefit of treatment in terms of physical function and everyday life activity would be valuable to inform physicians and help people with OP understand these potential benefits when making treatment decisions. This could, in turn, help to improve compliance and persistence with treatment. In contrast, studies of exercise often collect patient-centred outcomes [61]. However, it might be advisable to agree on a core set of outcomes for future trials to facilitate comparisons.

The findings of this review also provide insight on how communication between healthcare professionals and people with OP could be improved in terms of the information and advice provided to patients related to physical function and activity. Information and advice provided to people with OP are an important factor in how they will respond and persist with treatment. In order to improve these interactions, our suggestion to clinicians and other healthcare professionals is to provide clear, simple explanations about the physiology, symptoms and potential impacts of OP on physical function at diagnosis, as well as the possibility of social and psychological issues that may also affect physical function. Having this greater awareness of the long-term consequences of OP in terms of physical function, body shape and everyday activity may encourage persons with OP to better comply and persist with treatment, as these impacts may be more salient to patients than fracture. In addition, in order to minimise the confusion around exercise recommendations, physicians should provide evidence-based exercise guidelines rather than generic advice to exercise, to walk or get more active, and should make referrals to exercise advice from physical therapists or exercise physiologists specialising in the treatment of OP.

The findings suggest that further investigation and research into certain areas related to OP and physical function would be useful. One such area is further assessing the relationship between muscle and bone strength in OP and how they interact when it comes to limitations in physical function and performance. It may also be valuable to investigate further the relationship among reduced societal participation, physical function and psychological impact. In particular, this would enable us to understand the possible effects of a reduction in societal participation that can occur as a result of OP. We would also suggest studies to evaluate the optimal way to engage people with OP in an appropriate and effective exercise treatment plan.

Limitations of this report should be acknowledged. With regards to the literature review, a key limitation was the insufficient classification of sample characteristics and methods in the selected publications. Most importantly, many publications failed to state whether the study participants had a confirmed diagnosis of OP, and others included mixed samples of those with OP and/or those who had experienced fractures. While some studies did make comparisons between OP patients with and without fractures, results were reported in terms of greater impact among fracture subgroups, rather than presenting results to describe the level of physical function and life impact among OP patients with or without fracture. The secondary qualitative analysis of interview transcripts was limited by the original aim to develop a specific PRO instrument. As a result, although problems relating to physical function and other factors influencing OP-related impairment were captured during the interviews, they were rarely explored in detail. Participants were not asked further questions regarding why a specific activity was problematic or how they felt about any limitations caused by their OP. Furthermore, in cases where participants did not report limitations, it is unclear whether they did not experience them or simply had not mentioned them during the interviews. In addition, many of the participants had comorbidities that also could impact physical function making it sometimes difficult to attribute an impact on physical function or activity to OP versus a comorbid condition. To address this limitation, input was sought from clinical experts through a review process to confirm validity and inform interpretation of the information reported.

In summary, OP and fracture can have a profound impact on physical function and everyday activity, and this impact accumulates over time through a cycle of impairment, as fracture leads to longer term detriments in physical function, including loss of muscle, activity avoidance and reduced 
physical capacity, which in turn leads to greater risk of fracture and potentially further reductions in physical function. This cycle of impairment is complex, as other physical, psychosocial and treatment-related factors, such as comorbid conditions, fears and beliefs about physical activity and risk of fracture influence limitations in physical activity and everyday function. More information on how treatments impact patients in terms of physical function and everyday activity would benefit both healthcare professionals and persons with OP in making treatment decisions and improving overall outcomes. Furthermore, informing patients about the potential long-term physical consequences of OP and impact on everyday life activity may help improve compliance and persistence with treatment, as these impacts may be more salient to patients than fracture incidence.

Acknowledgements We would like to acknowledge the following people for their role in the development of this review article: Emuella Flood for input and review of the manuscript, Samuel Llewellyn for editorial review of the paper, April Naegeli and Russel Burge (Eli Lilly) for commissioning the work and their involvement in the literature review and secondary qualitative analysis and Sarah Corden and Monique Curran for editorial assistance in preparing the manuscript.

\section{Compliance with ethical standards}

Disclosure This work was completed as part of a programme of work in OP funded by Eli Lilly.

Conflicts of interest This review was funded by Eli Lilly, who commissioned ICON plc to conduct the work. Chloe Patel, Catherine Bottomley, Hayley M. de Freitas, Cicely Kerr and Sarah Shingler were employees of ICON plc at the time the research was completed. Deborah T. Gold, $\mathrm{PhD}$ is a consultant and speaker for Eli Lilly \& Co. and Amgen. Lora Giangregorio worked as a consultant for ICON plc on this research and has received funding for other research from Merck Canada Inc. (primary investigator) and Amgen (co-investigator). Susan Randall is employed by the National Osteoporosis Foundation.

Open Access This article is distributed under the terms of the Creative Commons Attribution-NonCommercial 4.0 International License (http:// creativecommons.org/licenses/by-nc/4.0/), which permits any noncommercial use, distribution, and reproduction in any medium, provided you give appropriate credit to the original author(s) and the source, provide a link to the Creative Commons license, and indicate if changes were made.

\section{References}

1. Reginster JY, Burlet N (2006) Osteoporosis: a still increasing prevalence. Bone 38(Suppl 1):S4-S9

2. Endocrine Society (2016) Endocrine facts and figures: bone and mineral. First edition. http://endocrinefacts.org/healthconditions/bone-calcium/2-osteoporosis/. Accessed 21 Mar 2016

3. National Osteoporosis Foundation (2016) What is osteoporosis and what causes it? https://www.nof.org/patients/what-is-osteoporosis/. Accessed 30 Nov 2016

4. World Health Organization (2004) WHO scientific group on the assessment of osteoporosis at primary health care level. Report of a WHO Study Group. http://www.who.int/chp/topics/Osteoporosis. pdf. Accessed 12 Oct 2015

5. U.S. Food and Drug Administration (1994) Guidelines for preclinical and clinical evaluation of agents used in the prevention or treatment of postmenopausal osteoporosis. http://www.fda. gov/downloads/UCM131206.pdf. Accessed 12 Oct 2015

6. Papaioannou A, Kennedy CC, Ioannidis G et al (2009) The impact of incident fractures on health-related quality of life: 5 years of data from the Canadian Multicentre Osteoporosis Study. Osteoporos Int 20:703-714

7. Oleksik AM, Ewing S, Shen W et al (2005) Impact of incident vertebral fractures on health related quality of life (HRQOL) in postmenopausal women with prevalent vertebral fractures. Osteoporos Int 16:861-870

8. Adachi JD, Ioannidis G, Olszynski WP et al (2002) The impact of incident vertebral and non-vertebral fractures on health related quality of life in postmenopausal women. BMC Musculoskelet Disord $3: 11$

9. Borgström F, Lekander I, Ivergård M et al (2013) The International Costs and Utilities Related to Osteoporotic Fractures Study (ICUROS) - quality of life during the first 4 months after fracture. Osteoporos Int 24:811-823

10. U.S. Food and Drug Administration (2009). Guidance for industrypatient-reported outcome measures: use in medical product development to support labeling claims. http://www.fda. gov/downloads/Drugs/Guidances/UCM193282.pdf. Accessed 12 Oct 2015

11. Patient-Centred Outcomes Research Institute (2015). www.pcori. org. Accessed 12 Oct 2015

12. Crans GG, Silverman SL, Genant HK et al (2004) Association of severe vertebral fractures with reduced quality of life: reduction in the incidence of severe vertebral fractures by terparatide. Arthritis Rheum 50:4028-4034

13. Marquis P, Roux C, De La Loge C et al (2008) Strontium ranelate prevents quality of life impairment in postmenopausal women with established vertebral osteoporosis. Osteoporos Int 19:503-510

14. Oleksik A, Lips P, Dawson A et al (2000) Health-related quality of life in postmenopausal women with low BMD with or without prevalent vertebral fractures. J Bone Miner Res 15:1384-1392

15. Silverman S, Viswanathan HN, Yang YC et al (2012) Impact of clinical fractures on health-related quality of life is dependent on time of assessment since fracture: results from the FREEDOM trial. Osteoporos Int 23:1361-1369

16. Naegeli AN, Nixon A, Burge R et al (2014) Development of the Osteoporosis Assessment Questionnaire-Physical Function (OPAQ-PF): an osteoporosis-targeted, patient-reported outcomes (PRO) measure of physical function. Osteoporos Int 25:579-588

17. Cress ME, Buchner DM, Questad KA et al (1996) Continuous scale physical functional performance in healthy older adults: a validation study. Arch Phys Med Rehabil 77:1243-1250

18. Delbaere K, Van den Noortgate N, Bourgois J et al (2006) The physical performance test as a predictor of frequent fallers: a prospective community-based cohort study. Clin Rehabil 20:83-90

19. MacIntyre NJ, Stavness CL, Adachi JD (2010) The safe functional motion test is reliable for assessment of functional movements in individuals at risk for osteoporotic fracture. Clin Rheumatol 29: $143-150$

20. Recknor CP, Grant SL, Recknor JC, Macintyre NJ (2013) Scores on the safe functional motion test are associated with prevalent fractures and fall history. Physiother Can 65:75-83

21. Cornwell EY, Waite LJ (2009) Social disconnectedness, perceived isolation, and health among older adults. J Health Soc Behav 50: $31-48$ 
22. Delmas PD, van de Langerit L, Watts NB et al (2005) Underdiagnosis of vertebral is a worldwide problem: the IMPACT study. J Bone Miner Res 20:557-563

23. Ross PD, Davis JW, Epstein RS, Wasnich RD (1991) Pre-existing fractures and bone mass predict vertebral fracture incidence in women. Ann Intern Med 114:919-923

24. Silverman SL, Minshall ME, Shen W et al (2001) The relationship of health-related quality of life to prevalent and incident vertebral fractures in postmenopausal women with osteoporosis: results from the Multiple Outcomes of Raloxifene Evaluation Study. Arthritis Rheum 44:2611-2619

25. Nevitt MC, Ettinger B, Black CM et al (1998) The association of radiographically detected vertebral fractures with back pain and function: a prospective study. Ann Intern Med 128:793-800

26. U.S. Department of Health and Human Services (1994) Bone health and osteoporosis: a report of the surgeon general. Available from: http://www.surgeongeneral.gov/library/bonehealth/docs/full_ report.pdf. Accessed 24 Nov 2015

27. Kado DM, Huang MH, Nguyen CB et al (2009) Hyperkyphosis predicts mortality independent of vertebral osteoporosis in older women. Ann Intern Med 150:681-687

28. Huang MH, Barrett-Connor E, Greendale GA, Kado DM (2006) Hyperkyphotic posture and risk of future osteoporotic fractures: the Rancho Bernardo study. J Bone Miner Res 21:419-423

29. Edwards MH, Dennison EM, Aihie Sayer A, Fielding R, Cooper C (2015) Osteoporosis and sarcopenia in older age. Bone 80:126-130

30. Edwards MH, Gregson CL, Patel HP et al (2013) Muscle size, strength, and physical performance and their associations with bone structure in the Hertfordshire Cohort Study. J Bone Miner Res 28: 2295-2304

31. Rikkonen T, Sirola J, Salovaara K et al (2012) Muscle strength and body composition are clinical indicators of osteoporosis. Calcif Tissue Int 91(2):131-138

32. Schipilow JD, Macdonald HM, Liphardta AM et al (2013) Bone micro-architecture, estimated bone strength, and the muscle-bone interaction in elite athletes: an HR-pQCT study. Bone 56:281-289

33. NIH Consensus Panel on Osteoporosis Prevention, Diagnosis, and Therapy (2001) Osteoporosis prevention, diagnosis, and therapy. JAMA 285:785-795

34. Gold DT, Shipp KM, Pieper CF et al (2004) Group treatment improves trunk strength and psychological status in older women with vertebral fractures: results of a randomized, clinical trial. J Am Geriatr Soc 52:1471-1478

35. Cook DJ, Guyatt GH, Adachi JD et al (1993) Quality of life issues in women with vertebral fractures due to osteoporosis. Arthritis Rheum 36:750-756

36. Hübscher M, Vogt L, Schmidt K et al (2010) Perceived pain, fear of falling and physical function in women with osteoporosis. Gait Posture 32:383-385

37. Gold DT (2001) The non-skeletal consequences of osteoporotic fractures. Psychological and social outcomes. Rheum Dis Clin N Am 27:255-262

38. Gold DT, Solimeo S (2006) Osteoporosis and depression: a historical perspective. Curr Osteoporos Rep 4:134-139

39. Piscitelli P, Metozzi A, Benvenuti E et al (2012) Connections between the outcomes of osteoporotic hip fractures and depression, delirium or dementia in elderly patients: rationale and preliminary data from the CODE study. Clin Cases Miner Bone Metab 9:40-44

40. Chang CY, Chen WL, Liou YF et al (2014) Increased risk of major depression in the three years following a femoral neck fracture - a national population-based follow-up study. PLoS One 9:e89867

41. Bianchi ML, Orsini MR, Saraifoger S et al (2005) Quality of life in post-menopausal osteoporosis. Health Qual Life Outcomes 3:78
42. Courtin, Knapp (2015) Social isolation, loneliness and health in old age: a scoping review. Health Soc Care Community. doi:10.1111 /hsc. 12311

43. Choices program: for better bone health. Available from: https://ag. tennessee.edu/fcs/Documents/BetterBoneHealth.pdf. Accessed 12 Oct 2015

44. Gold DT, Silverman SL (2004) Osteoporosis self-management: choices for better bone health. South Med J 97:551-554

45. Nuño-Solinis R, Rodríguez-Pereira C, Alonso-Morán E et al (2014) Comorbidity and healthcare expenditure in women with osteoporosis living in the Basque country (Spain). J Osteoporos 2014:205954

46. Gregson CL, Dennison EM, Compston JE et al (2014) Diseasespecific perception of fracture risk and incident fracture rates: GLOW cohort study. Osteoporos Int 25:85-95

47. Mathews AE, Laditka SB, Ladtika SW et al (2010) Older adult's perceived physical activity enablers and barriers: a multicultural perspective. J Aging Phys Act 18:119-140

48. Giangregorio L, Dolovich L, Cranney A et al (2009) Osteoporosis risk perceptions among patients who have sustained a fragility fracture. Patient Educ Couns 74:213-220

49. Meadows LM, Mrkonjic LA, Petersen KM, Lagendyk LE (2004) After the fall: women's views of fractures in relation to bone health at midlife. Women Health 39:47-62

50. Meadows LM, Mrkonjic LA (2003) Breaking-bad news: women's experiences of fractures at midlife. Can J Public Health 94:427-430

51. Barker M, D'Angelo S, Ntani G et al (2016) The relationship between maternal self-efficacy, compliance and outcome in a trial of vitamin D supplementation in pregnancy. Osteoporos Int doi:10.1007/s00198-016-3721-5

52. Sinaki M, Mikkelsen BA (1986) Relationship between bone mineral density of spine and strength of back extensors in healthy postmenopausal women. Mayo Clin Proc 61:116-122

53. Giangregorio LM, MacIntyre NJ, Heinonen A (2014) Too fit to fracture: a consensus on future research priorities in osteoporosis and exercise. Osteoporos Int 25:1465-1472

54. Papaioannou A, Watts NB, Kendler DL (2002) Diagnosis and management of vertebral fractures in elderly adults. Am J Med 113: 220-228

55. Silverman S, Calderon A, Kaw K et al (2013) Patient weighting of osteoporosis medication attributes across racial and ethnic groups: a study of osteoporosis medication preferences using conjoint analysis. Osteoporos Int 24:2067-2077

56. Silverman SL, Schousboe JT, Gold DT (2011) Oral bisphosphonate compliance and persistence: a matter of choice? Osteoporos Int 22: 21-26

57. Howe TE, Shea B, Dawson LJ et al (2011) Exercise for preventing and treating osteoporosis in postmenopausal women. Cochrane Database Syst Rev 6:CD000333

58. Prince R, Devine A, Dick I (1995) The effects of calcium supplementation (milk powder or tablets) and exercise on one density in postmenopausal women. J Bone Miner Res 10:1068-1075

59. Bocalini DS, Serra AJ, Dos Santos L et al (2009) Strength training preserves the bone mineral density of postmenopausal women without hormone replacement therapy. J Aging Health 21:519-527

60. Baert V, Gorus E, Mets T, Bautmans I (2015) Motivators and barriers for physical activity in older adults with osteoporosis. J Geriatr Phys Ther 38:105-114

61. Giangregorio LM, Macintyre NJ, Thabane L et al (2013) Exercise for improving outcomes after osteoporotic vertebral fracture. Cochrane Database Syst Rev 1:CD008618 